\title{
Elevated Angiotensin II in Rat Nodose Ganglia Primes Diabetes-Blunted Arterial Baroreflex Sensitivity: Involvement of NADPH Oxidase-Derived Superoxide
}

\section{Yu-Long Li}

Department of Emergency Medicine, University of Nebraska Medical Center, Omaha, NE 68198, USA

\begin{abstract}
Clinical trials and experimental animal studies have confirmed the contribution of arterial baroreflex impairment in causing excess morbidity and mortality in type-1 diabetes. Our previous study has shown that angiotensin II (Ang II)-NADPH oxidase-superoxide signaling is associated with the reduced cell excitability in the aortic baroreceptor neurons (a primary afferent limb of the arterial baroreflex) from diabetic rats. In this study, we examined whether above-mentioned signaling might contribute to the blunted baroreflex sensitivity in streptozotocin-induced diabetic rats. Using Ang II ${ }^{125}$ radioimmunoassay and lucigeninchemiluminescence method, we found Ang II concentration, NADPH oxidase activity, and superoxide production in the nodose ganglia were enhanced in diabetic rats, compared to sham rats.As an index of the arterial baroreflex sensitivity, the reflex decreases in blood pressure and heart rate evoked by unilateral steady-frequency aortic depressor nerve stimulation were attenuated in diabetic rats. Local microinjection $(50 \mathrm{nl})$ of losartan (an AT, receptor antagonist, $1 \mathrm{nmol}$ ), apocynin (a NADPH oxidase inhibitor, $1 \mathrm{nmol}$ ), and tempol (a superoxide dismutase mimetic, $10 \mathrm{nmol}$ ) into the nodose ganglia significantly improved the arterial baroreflex sensitivity in diabetic rats. In addition, these three chemicals also normalized exogenous Ang II-attenuated arterial baroreflex sensitivity in sham rats. These results indicate that overactivation of the Ang II-NADPH oxidase-superoxide signal pathway in the nodose ganglia contributes to the blunted baroreflex sensitivity in diabetes.
\end{abstract}

Keywords: Angiotensin II; Baroreflex; Diabetes; NADPH oxidase; Superoxide

\section{Introduction}

Arterial baroreflex is a homeostatic mechanism that normally alters heart rate and blood pressure in response to changes in arterial wall tension detected by the arterial baroreceptors in the carotid sinus and aortic arch. Much evidence has shown that the arterial baroreflex sensitivity is attenuated in type 1 diabetic patients and animal models [1-7], which may contribute to the enhanced morbidity and mortality in diabetic patients $[8,9]$. Although the mechanisms responsible for the attenuated arterial baroreflex function in the type 1 diabetes are not yet fully understood, any part of the arterial baroreflex arc can contribute to the arterial baroreflex dysfunction in the type 1 diabetes. Aortic baroreceptor neurons contained within the nodose ganglia serve as the primary afferent limb of the arterial baroreflex. One recent study has shown that the impaired baroreflex sensitivity correlates with the changes in the nucleus tractus solitarii neural firing rates, which is an effect of changes in afferent nervous behavior and in the nucleus tractus solitarii cells themselves in the streptozotocin-induced diabetic rats [10]. Fazan, et al. [11] have found that the morphological change in aortic depressor nerve, an afferent arm of the baroreflex may result in the baroreflex impairment in the streptozotocin-induced diabetic rats. Our previous study has shown that the cell excitability of the aortic baroreceptor neurons is blunted in the streptozotocin-induced diabetic rats [12]. However, until now there is no direct evidence to clarify that the depressed aortic baroreceptor neuron excitability can be involved in the impairment of the baroreflex sensitivity in type 1 diabetes.

Angiotensin II (Ang II, an endogenous peptide) in plasma and local tissues is elevated in human and animals with diabetes [13-16]. Patel et al. have reported that elevation of the Ang II in the paraventricular nucleus induces the increase of sympathoexcitation in diabetes through triggering the superoxide production [17]. Our previous studies have demonstrated that elevation of local Ang II level in the nodose ganglia can blunt the membrane excitability of the aortic baroreceptor neurons via NADPH oxidase-derived superoxide in the type 1 diabetes $[14,18]$. Therefore, we investigated whether local microinjection of Ang II type $1\left(\mathrm{AT}_{1}\right)$ receptor antagonist (losartan), NADPH oxidase inhibitor (apocynin), and superoxide dismutase (SOD) mimetic (tempol) into the nodose ganglia could improve the arterial baroreflex function in the streptozotocin (STZ)-induced diabetic rats. We also measured the endogenous Ang II concentration, NADPH oxidase activity, and superoxide production in the nodose ganglia from sham and STZinduced diabetic rats.

\section{Materials and Methods}

Male Sprague-Dawley rats (200-220 g) were housed two per cage under controlled temperature and humidity and a 12:12-h dark-light cycle. Water and rat chow were provided ad libitum. Experiments were approved by the University of Nebraska Medical Center Institutional Animal Care and Use Committee and were carried out in accordance with the National Institutes of Health (NIH Publication No. 85-23, revised 1996) and the American Physiological Society's Guides for the Care and Use of Laboratory Animals.

\section{Materials}

Losartan was a gift from Merck Co., NJ, USA. Apocynin was

Corresponding author: Yu-Long Li, M.D., Ph.D., Department of Emergency Medicine, University of Nebraska Medical Center, Omaha, NE 68198-5850, USA, Tel: 1-402-559-3016; Fax: 1-402-559-9659; E-mail: yulongli@unmc.edu Received July 29, 2011; Accepted August 28, 2011; Published September 08 2011

Citation: Li Y (2011) Elevated Angiotensin II in Rat Nodose Ganglia Primes Diabetes-Blunted Arterial Baroreflex Sensitivity: Involvement of NADPH OxidaseDerived Superoxide. J Diabetes Metab 2:135. doi:10.4172/2155-6156.1000135

Copyright: ( $2011 \mathrm{Li} \mathrm{Y}$. This is an open-access article distributed under the terms of the Creative Commons Attribution License, which permits unrestricted use, distribution, and reproduction in any medium, provided the original author and source are credited. 
purchased from CalBiochem, CA, USA. Tempol was purchased from Alexis Biochemicals Co., CA, USA. Other chemicals used in this study were obtained from Sigma-Aldrich Chemical Co., MO, USA.

\section{Induction of diabetes}

Rats were randomly assigned to sham and diabetic rats. Diabetes was induced by a single intraperitoneal injection of streptozotocin $(65$ $\mathrm{mg} / \mathrm{kg}$, Sigma) in a $2 \%$ solution of $0.1 \mathrm{M}$ cold citrate buffer as previously described $(12,19)$. Sham rats received a similar injection of vehicle. Diabetes was identified by polydipsia, polyuria, and blood glucose $>$ $250 \mathrm{mg} / \mathrm{dl}$ (Accu-check Aviva, Boehringer Mannheim). Rats receiving streptozotocin but with blood glucose $<250 \mathrm{mg} / \mathrm{dl}$ were excluded from study. Blood glucose and body weight in all rats were measured weekly. All experiments were taken at 6-8 weeks after single dose injection of streptozotocin or vehicle. On the day of the terminal experiment, the rat was anaesthetized with a combination of urethane $(800 \mathrm{mg} / \mathrm{kg}$, i.p. $)$ and chloralose ( $40 \mathrm{mg} / \mathrm{kg}$, i.p.). Then, in one part of the experiments, aortic baroreflex sensitivity was measured (see below). In another part of the experiments, nodose ganglia in each rat were acutely removed for varied measurements.

\section{Ang II measurement}

Plasma was prepared from arterial blood. Ang II concentration in plasma and nodose ganglia was measured by Ang II ${ }^{125} \mathrm{I}$ radioimmunoassay kit (Buhlmann Laboratories, Switzerland). The final Ang II concentration was counted by 1470 Automatic Gamma Counter (Perkin Elmer, Shelton, CT) and calculated with a standard curve generated for each experiment.

\section{Measurement of NADPH oxidase activity and superoxide production}

Nodose ganglion samples were homogenized in PBS solution at $4^{\circ} \mathrm{C}$ and continuously kept at $4^{\circ} \mathrm{C}$ until the measurement of NADPH oxidase activity and superoxide production to prevent the protein degradation of NADPH oxidase. Total protein concentration was determined using a bicinchoninic acid protein assay kit (Pierce; Rockford, IL). Superoxide ion production was measured using lucigenin chemiluminescence method as described previously [20-22]. The homogenate $(0.3 \mathrm{ml})$ was placed in $0.5 \mathrm{ml}$ microfuge containing dark-adapted lucigenin $(5 \mu \mathrm{M})$, and then accumulative light emission was recorded for $5 \mathrm{~min}$ in a TD20/20 Luminometer (Turner Designs, Sunnyvale, CA). When enough NADPH $(100 \mu \mathrm{M}$, a substrate of NADPH oxidase) was added in homogenate, the accumulative light emission was recorded for $5 \mathrm{~min}$, which was served as NADPH oxidase activity [23].

\section{Recording of aortic baroreflex sensitivity [24]}

The rat is a useful animal model for the study of baroreflex sensitivity because rat aortic depressor nerve contains only baroreceptor afferent fibers and no chemoreceptor afferent fibers to transmit the chemoreceptor information [25-28].

Rat was anesthetized with a combination of urethane $\left(800 \mathrm{mg} \mathrm{kg}^{-1}\right.$, I.P.) and chloralose $\left(80 \mathrm{mg} \mathrm{kg}^{-1}\right.$, I.P.), with supplements of chloralose (10 $\mathrm{mg} \mathrm{kg}^{-1}$, I.P.) per $2 \mathrm{~h}$. After a ventral midline incision was made in the neck, the trachea was cannulated, and rat breathed spontaneously. Catheters were implanted into the femoral artery for arterial blood pressure and heart rate measurements. The blood pressure and heart rate were recorded by LabChart 6 (ADInstruments, Colorado Springs, $\mathrm{CO})$

Left or right aortic depressor nerve was isolated for 3-5 mm near its junction with the superior laryngeal nerve. The nerve was placed on the stimulating electrodes (bipolar) and covered with mineral oil. All other nerves were intact. Unilateral aortic depressor nerve stimulation was imposed using $10 \mathrm{~s}$ of constant-frequency stimulation with 0.1 ms pulse duration and intensity of $18 \mathrm{~V}, 1-100 \mathrm{~Hz}$ (activating A- and C-type) [24]. Reflex changes in blood pressure and heart rate related to different stimulating parameters were used as the indices of baroreflex sensitivity for the A- and C-type baroreflex. The effect of AT receptor antagonist, NADPH oxidase inhibitor, and SOD mimetic on the aortic baroreflex sensitivity was measured after each of these chemicals (50 nl) was microinjected into unilateral nodose ganglion using a glass micropipette.

\section{Statistical analysis}

All data are presented as means \pm SE. SigmaStat 3.5 was used for data analysis. Statistical significance was determined by student's unpaired $\mathrm{t}$ test for body weight, blood glucose, Ang II concentration, NADPH oxidase activity, and superoxide production. A two-way ANOVA, with a Bonferroni procedure for post hoc was used in comparisons of aortic baroreflex sensitivity in various treatments. Statistical significance was accepted when $\mathrm{p}<0.05$.

\section{Results}

\section{General data}

After 6-8 weeks of STZ injection (65 mg/kg, i.p.), the mean blood glucose in the diabetic group was significantly increased to $436 \pm 17$ $\mathrm{mg} / \mathrm{dl}$, compared with that in the sham group $(101 \pm 13 \mathrm{mg} / \mathrm{dl}, \mathrm{p}<0.05)$. The body weight and heart rate in the diabetic rats $(228 \pm 22 \mathrm{~g}$ and 334 \pm 21 beats/min) were lower than those in the sham rats $(372 \pm 18 \mathrm{~g}$ and $365 \pm 15$ beats/min, $\mathrm{p}<0.05)$. There was no significant difference in blood pressure between the sham and the diabetic groups (92 \pm 8 and $89 \pm 10 \mathrm{~mm} \mathrm{Hg}$ ).
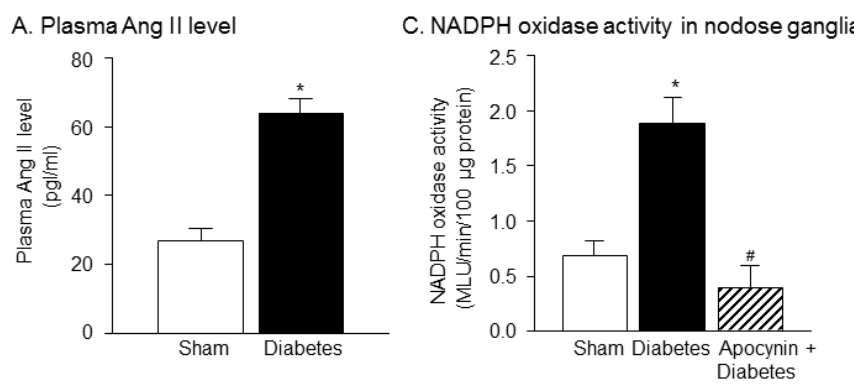

B. Ang II level in nodose ganglia
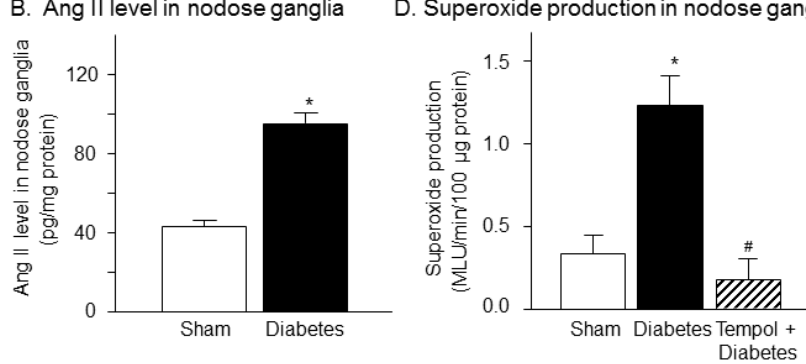

Figure 1: Angiotensin II concentration, NADPH oxidase activity, and superoxide production in sham and STZ-induced diabetic rats. Apocynin (a NADPH oxidase inhibitor, $100 \mu \mathrm{M}$ ) and tempol (a superoxide dismutase mimetic, $1 \mathrm{mM}$ ) were used to confirm the specificity of the measurement for NADPH oxidase and superoxide production respectively. Data are mean \pm SE $n=6$ rats in each group. ${ }^{*} P<0.05$ vs. sham rats; ${ }^{*} p<0.05$ vs. diabetes. 


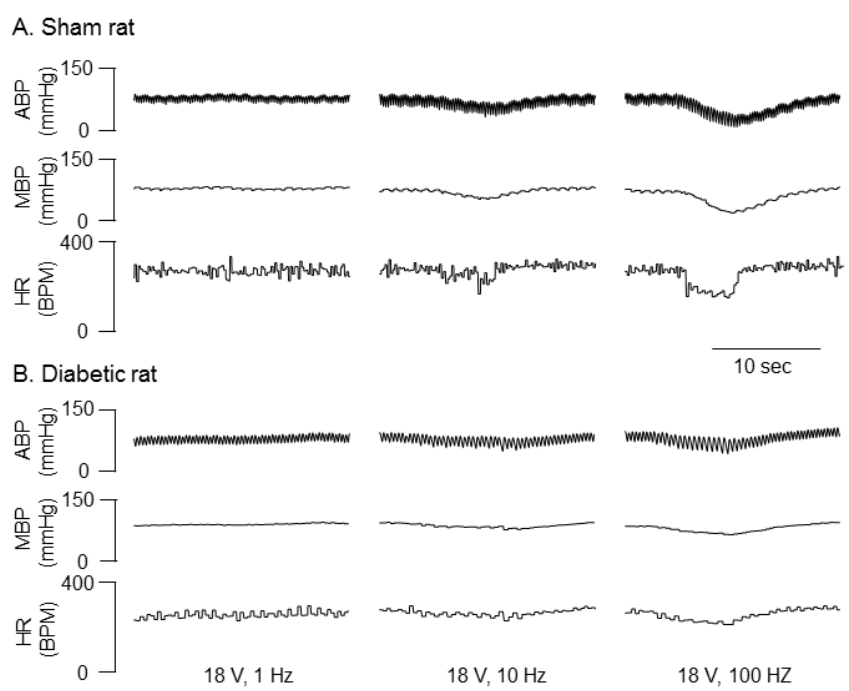

Figure 2: Representative tracings of blood pressure and heart rate responses to aortic depressor nerve (ADN) stimulation in sham and STZ-induced diabetic rats.ABP, arterial blood pressure; MBP, mean blood pressure; HR, heart rate.
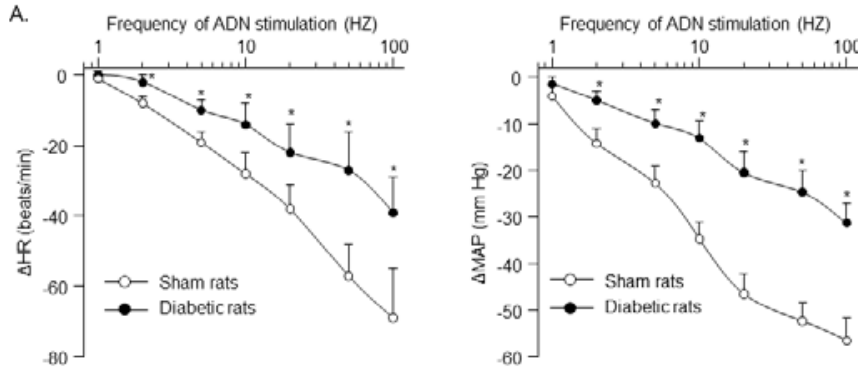

B.
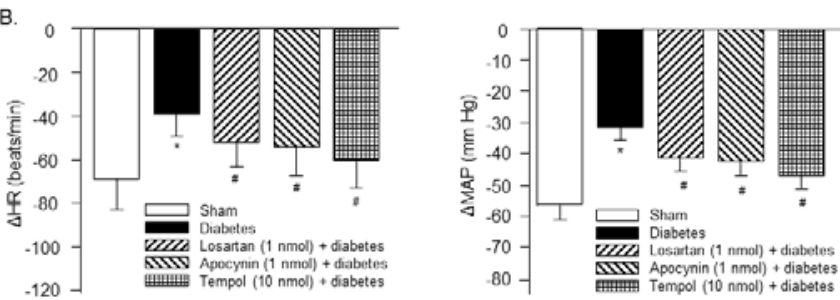

Figure 3: Effects of losartan, apocynin, and tempol on the arterial baroreflex sensitivity in STZ-induced diabetic rats. $\mathrm{A}$, reflex $\triangle \mathrm{MAP}$ and $\triangle \mathrm{HR}$ in response to different frequencies of $A D N$ stimulation in sham and diabetic rats. $B$, reflex $\triangle M A P$ and $\triangle \mathrm{HR}$ in response to ADN stimulation $(100 \mathrm{~Hz})$ in each group (chemicals were microinjected into the nodose ganglion). Data are mean \pm $\mathrm{SE}, \mathrm{n}=6$ rats in each group. ${ }^{*} \mathrm{P}<0.05$ vs. sham rats; ${ }^{*} \mathrm{p}<0.05$ vs. diabetic rats.

Ang II concentration, NADPH oxidase activity, and superoxide production in the sham and the diabetic rats

The Ang II concentration in the plasma and local nodose ganglion tissue from diabetic rats was significantly elevated, compared to those from sham rats (Figure 1A and 1B). Similarly, diabetes also increased the NADPH oxidase activity and superoxide production in the nodose ganglia (Figure $1 \mathrm{C}$ and $1 \mathrm{D}, \mathrm{p}<0.05$ vs. sham rats). Additionally, apocynin (a NADPH oxidase inhibitor, $100 \mu \mathrm{M}$ ) significantly inhibited the diabetes-enhanced NADPH oxidase activity; and tempol (a SOD mimetic, $1 \mathrm{mM}$ ) markedly scavenged the superoxide production in diabetes (Figure 1C and 1D), which confirmed the specificity of NADPH oxidase and superoxide production measured in the method.
Effect of losartan, apocynin, and tempol on the aortic baroreflex sensitivity in diabetic rats

Reflex changes in blood pressure and heart rate related to different electrical stimulation of aortic depressor nerve were used as the indices of the aortic baroreflex sensitivity. Figure 2 illustrates typical recording of the blood pressure and heart rate in some electrical stimulating frequencies in sham and diabetic rats. From mean data shown in Figure $3 \mathrm{~A}$, reflex decreases in blood pressure and heart rate evoked by unilateral, steady-frequency stimulation of the aortic depressor nerve, were attenuated in anesthetized diabetic rats compared with sham rats. Local application $(50 \mathrm{nl})$ of losartan (an $\mathrm{AT}_{1}$ receptor antagonist, 1 nmol), apocynin (a NADPH oxidase inhibitor, $1 \mathrm{nmol}$ ), or tempol (a SOD mimetic, $10 \mathrm{nmol}$ ) into the nodose ganglion markedly improved the responses of blood pressure and heart rate to the aortic depressor nerve stimulation $(100 \mathrm{~Hz})$ in diabetic rats. However, these reflex responses to the aortic depressor nerve stimulation $(100 \mathrm{~Hz})$ were not recovered to the level seen in sham rats (Figure 3B).

Effect of Ang II on the aortic baroreflex sensitivity in sham rats

To further clarify whether endogenous Ang II is involved in the attenuated aortic baroreflex sensitivity in diabetic rats, I investigated the effect of Ang II on the aortic baroreflex sensitivity in sham rats. Local application of Ang II $(0.2 \mathrm{nmol})$ into the nodose ganglion mimicked the diabetes to decrease the responses of blood pressure and heart rate to the aortic depressor nerve stimulation in sham rats (Figure $4 \mathrm{~A})$. Although losartan (1 nmol), apocynin (1 nmol), or tempol (10 nmol) alone (without Ang II) did not show any effect on the aortic baroreflex sensitivity, these chemicals totally normalized the inhibitory effect of Ang II on the aortic baroreflex sensitivity (Figure 4B).

\section{Discussion}

The present study demonstrates that: 1) diabetes blunted the aortic
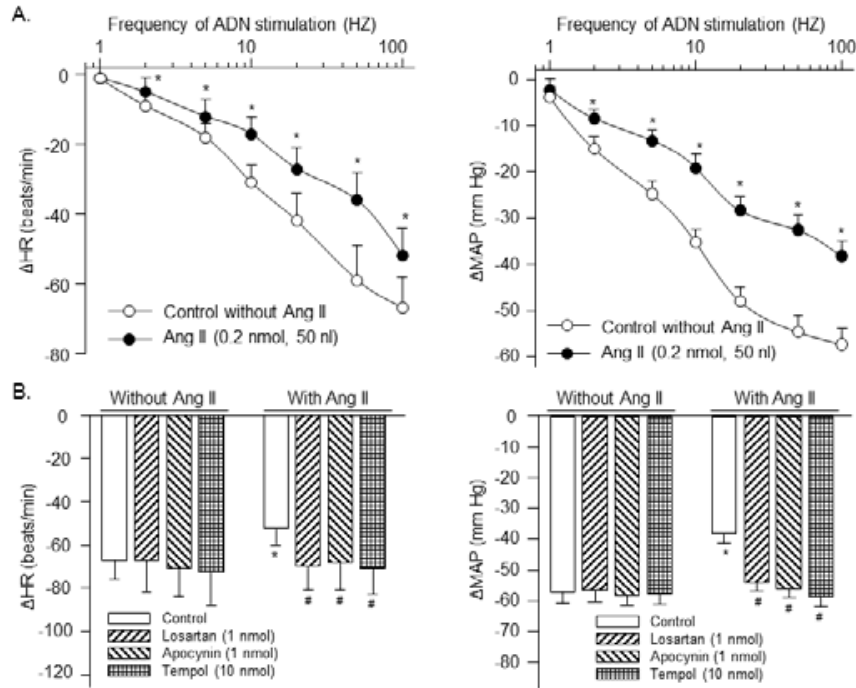

Figure 4: Effects of exogenous Ang II on the arterial baroreflex sensitivity in sham rats. $A$, reflex $\triangle M A P$ and $\triangle H R$ in response to different frequencies of ADN stimulation before and after local microinjection of Ang II into the nodose ganglia in sham rats. $B$, reflex $\triangle M A P$ and $\triangle H R$ in response to $A D N$ stimulation $(100 \mathrm{~Hz})$ in each group. Data are mean $\pm S E, n=6$ rats in each group. ${ }^{*} \mathrm{P}<0.05$ vs. control without Ang II; $" \mathrm{p}<0.05$ vs. control with Ang II. 
baroreflex sensitivity; 2) local microinjection of losartan (a selective $\mathrm{AT}_{1}$ receptor antagonist), apocynin (a NADPH oxidase inhibitor), and tempol (a SOD mimetic) into the nodose ganglion partially improved the aortic baroreflex sensitivity in STZ-induced diabetic rats; 3) losartan, apocynin, and tempol also fully normalized exogenous Ang II-attenuated aortic baroreflex sensitivity in sham rats; 4) Ang II concentration in plasma and nodose ganglia, NADPH oxidase activity, and superoxide production were increased in the diabetic rats. These results indicate that over-excited endogenous Ang II-NADPH oxidasesuperoxide signaling in the nodose ganglia is involved in the attenuated aortic baroreflex sensitivity in the diabetic state.

Arterial baroreflex contributes to regulating the blood pressure and heart rate by acting on both sympathetic and parasympathetic efferent limbs of the cardiovascular autonomic nervous system and maintaining the enough blood flow into all organs. Previous studies have shown that the arterial baroreflex dysfunction occurs in type-1 diabetic patients and experimental animal models [1-7]. Diabetic patients with the impairment of the arterial baroreflex have a higher mortality rate than those without normal arterial baroreflex function [8]. However, the mechanisms for the attenuated arterial baroreflex are still unclear. Baroreceptors (the primary afferent limb of the arterial baroreflex arc) sense the systemic blood pressure by baroreceptor terminals innervating aortic arch and carotid sinus and then transmit the arterial baroreceptor afferent discharge into the dorsal medial nucleus tractus solitarii (NTS, the first site of baroreceptor contacting with central nervous system), in which the integrated input signal elicits parasympathetic activation and sympathetic inhibition with subsequent decreases in peripheral vascular resistance, heart rate, cardiac contractility, and arterial blood pressure [29]. In general, the process of translating changes in arterial wall tension into impulse traffic to the nucleus tractus solitarii involves two broad functional steps: 1) mechanotransduction which is governed by the properties of mechanosensitive ion channels in the nerve terminal and the mechanical properties of the coupling of the arterial wall to the sensory terminal; and 2) spike initiation which is governed by the excitability of membrane voltage sensitive ion channels that influence the electrical (cable) properties of the axonal projection and cell body. In the present study, the reflex decreases of blood pressure and heart rate to the electrical stimulation of the aortic depressor nerve are markedly attenuated in STZ-induced diabetic rats (Figure 2 and 3). In addition, local microinjection of Ang II into the nodose ganglion could mimic the diabetes to decrease the arterial baroreflex sensitivity (Figure 4), and losartan (an $\mathrm{AT}_{1}$ receptor antagonist) significantly improved diabetes- and Ang II-induced the blunted arterial baroreflex sensitivity (Figure 3 and 4). These data provide the direct evidence that alteration of the baroreceptor function contributes to the attenuated arterial baroreflex sensitivity in STZ-induced diabetic rats.

Much evidence has indicated that Ang II-NADPH oxidasesuperoxide signaling may be a potential candidate in the regulation of the arterial baroreflex in diabetes. Ang II binds with $\mathrm{AT}_{1}$ receptors to activate NADPH oxidase, and the latter is thought to be a main source of the intracellular superoxide in many tissues including nodose ganglia [18,21,30-35]. In the present study, Ang II concentration, NADPH oxidase activity and superoxide production in the nodose ganglia were enhanced in STZ-diabetic rats (Figure 1). More importantly, losartan, apocynin, and tempol partially recovered the arterial baroreflex sensitivity in STZ-diabetic rats (Figure 3). Additionally, exogenous Ang II, like the diabetes, decreased the arterial baroreflex sensitivity in sham rats through the $\mathrm{AT}_{1}$ receptor- $\mathrm{NADPH}$ oxidase-derived superoxide because losartan, apocynin, and tempol also normalized the effect of exogenous Ang II on the arterial baroreflex sensitivity in sham rats (Figure 4). Our previous studies have shown that endogenous Ang II-NADPH oxidase-superoxide signaling is involved in diabetesattenuated aortic baroreceptor neuron excitability [18]. Based on the above results, we believe that endogenous Ang II-NADPH oxidasesuperoxide signaling contributes to the arterial baroreflex dysfunction in the type 1 diabetes mellitus via depressing the aortic baroreceptor neuron excitability.

Our present study also found that the plasma Ang II concentration was elevated in STZ-induced diabetic rats (Figure 1). In addition, local microinjection of losartan, apocynin, and tempol into the nodose ganglion partially improved the diabetes-attenuated arterial baroreflex sensitivity (Figure 3). It is possible that Ang II-NADPH oxidasesuperoxide may also affect other site(s) of the arterial baroreflex arc to blunt the arterial baroreflex in the diabetic state (such as central neural areas because enhanced Ang II-NADPH oxidase-superoxide signal is found in the paraventricular nucleus from STZ-induced diabetic rats [17]). It will be clarified in further study.

In conclusion, endogenous Ang II-NADPH oxidase-superoxide signaling is over-activated in the nodose ganglia, which contributes to the attenuated arterial baroreflex function in the diabetes. The present study provides new information on the mechanisms underlying the impaired arterial baroreflex in the type 1 diabetes and unveils important pharmacological targets for improving the arterial baroreflex function and reducing the mortality in the type 1 diabetes.

\section{Acknowledgments}

Author thanks Phyllis M. Anding for her technical assistance. This study was supported in part by a Scientist Development Grant 0730108N from American Heart Association National Center (to Y.L. Li) and by National Institute of Health Grant R01 HL-098503 (to Y.L. Li).

\section{References}

1. Bennett T, Farquhar IK, Hosking DJ, Hampton JR (1978) Assessment of methods for estimating autonomic nervous control of the heart in patients with diabetes mellitus. Diabetes 27: 1167-1174.

2. Dall'Ago P, Fernandes TG, Machado UF, Bello AA, Irigoyen MC (1997) Baroreflex and chemoreflex dysfunction in streptozotocin-diabetic rats. Braz $\mathrm{J}$ Med Biol Res 30: 119-124.

3. De Angelis KL, Oliveira AR, Dall'Ago P, Peixoto LR, Gadonski G, et al. (2000) Effects of exercise training on autonomic and myocardial dysfunction in streptozotocin-diabetic rats. Braz J Med Biol Res 33: 635-641.

4. Frattola A, Parati G, Gamba P, Paleari F, Mauri G, et al. (1997) Time and frequency domain estimates of spontaneous baroreflex sensitivity provide early detection of autonomic dysfunction in diabetes mellitus. Diabetologia 40: 14701475.

5. Lishner M, Akselrod S, Avi VM, Oz O, Divon M, et al. (1987) Spectral analysis of heart rate fluctuations. A non-invasive, sensitive method for the early diagnosis of autonomic neuropathy in diabetes mellitus. J Auton Nerv Syst 19: 119-125.

6. Maeda CY, Fernandes TG, Timm HB, Irigoyen MC (1995) Autonomic dysfunction in short-term experimental diabetes. Hypertension 26: 1100-1104.

7. Wegner JA, Lund DD, Overton JM, Edwards JG, Oda RP, et al. (1987) Select cardiovascular and metabolic responses of diabetic rats to moderate exercise training. Med Sci Sports Exerc 19: 497-503.

8. Ewing DJ, Campbell IW, Clarke BF (1980) The natural history of diabetic autonomic neuropathy. Q J Med 49: 95-108

9. Singh JP, Larson MG, O'Donnell CJ, Wilson PF, Tsuji H, et al. (2000) Association of hyperglycemia with reduced heart rate variability (The Framingham Heart Study). Am J Cardiol 86: 309-312.

10. Chen HY, Wu JS, Chen JJ, Cheng JT (2008) Impaired regulation function in cardiovascular neurons of nucleus tractus solitarii in streptozotocin-induced diabetic rats. Neurosci Lett 431: 161-166. 
Citation: Li Y (2011) Elevated Angiotensin II in Rat Nodose Ganglia Primes Diabetes-Blunted Arterial Baroreflex Sensitivity: Involvement of NADPH Oxidase-Derived Superoxide. J Diabetes Metab 2:135. doi:10.4172/2155-6156.1000135

Page 5 of 5

11. Fazan VP, Salgado HC, Barreira AA (2006) Aortic depressor nerve myelinated fibers in acute and chronic experimental diabetes. Am J Hypertens 19: 153160

12. Li YL, Tran TP, Muelleman R, Schultz HD (2008) Blunted excitability of aortic baroreceptor neurons in diabetic rats: involvement of hyperpolarizationactivated channel. Cardiovasc Res 79: 715-721.

13. Frustaci A, Kajstura J, Chimenti C, Jakoniuk I, Leri A, et al. (2000) Myocardial cell death in human diabetes. Circ Res 87: 1123-1132.

14. Li YL, Tran TP, Muelleman R, Schultz HD (2008) Elevated angiotensin II in rat nodose ganglia mediates diabetes-blunted arterial baroreflex sensitivity. Circulation 118: S360.

15. Sechi LA, Griffin CA, Schambelan M (1994) The cardiac renin-angiotensin system in STZ-induced diabetes. Diabetes 43: 1180-1184.

16. Shimoni Y, Liu XF (2004) Gender differences in ANG II levels and action on multiple $\mathrm{K}+$ current modulation pathways in diabetic rats. Am J Physiol Heart Circ Physiol 287: H311-H319.

17. Patel KP, Mayhan WG, Bidasee KR, Zheng H (2011) Enhanced angiotensin II-mediated central sympathoexcitation in streptozotocin-induced diabetes: role of superoxide anion. Am J Physiol Regul Integr Comp Physiol 300: R311-R320.

18. Zhang L, Tran TP, Muelleman RL, Li YL (2009) Involvement of NADPH oxidase-derived superoxide anion in diabetes-blunted aortic baroreceptor neuron excitability. FASEB J 28: 785.2.

19. Tu H, Zhang L, Tran TP, Muelleman RL, Li YL (2009) Diabetes alters protein expression of hyperpolarization-activated cyclic nucleotide-gated channel subunits in rat nodose ganglion cells. Neuroscience 165: 39-52.

20. Li JM, Shah AM (2003) Mechanism of endothelial cell NADPH oxidase activation by angiotensin II. Role of the p47phox subunit. J Biol Chem 278: 12094-12100.

21. Li YL, Gao L, Zucker IH, Schultz HD (2007) NADPH oxidase-derived superoxide anion mediates angiotensin II-enhanced carotid body chemoreceptor sensitivity in heart failure rabbits. Cardiovasc Res 75: 546-554.

22. Zhang L, Tu H, Li YL (2010) Angiotensin II enhances hyperpolarization-activated currents in rat aortic baroreceptor neurons: involvement of superoxide. Am J Physiol Cell Physiol 298: C98-C106.
23. Li JM, Shah AM (2002) Intracellular localization and preassembly of the NADPH oxidase complex in cultured endothelial cells. J Biol Chem 277: 19952-19960.

24. Fan W, Schild JH, Andresen MC (1999) Graded and dynamic reflex summation of myelinated and unmyelinated rat aortic baroreceptors. Am J Physiol 277 R748-R756.

25. Fan W, Reynolds PJ, Andresen MC (1996) Baroreflex frequency-response characteristics to aortic depressor and carotid sinus nerve stimulation in rats. Am J Physiol 271: H2218-H2227.

26. Kobayashi M, Cheng ZB, Tanaka K, Nosaka S (1999) Is the aortic depresso nerve involved in arterial chemoreflexes in rats? J Auton Nerv Syst 78: 38-48.

27. Sapru HN, Krieger AJ (1977) Carotid and aortic chemoreceptor function in the rat. J Appl Physiol 42: 344-348.

28. Sapru HN, Gonzalez E, Krieger AJ (1981) Aortic nerve stimulation in the rat cardiovascular and respiratory responses. Brain Res Bull 6: 393-398.

29. Lanfranchi PA, Somers VK (2002) Arterial baroreflex function and cardiovascular variability: interactions and implications. Am J Physiol Regul Integr Comp Physiol 283: R815-R826.

30. Cifuentes ME, Rey FE, Carretero OA, Pagano PJ (2000) Upregulation of p67(phox) and gp91(phox) in aortas from angiotensin Il-infused mice. Am J Physiol Heart Circ Physiol 279: H2234-H2240.

31. Franco MC, Akamine EH, Di Marco GS, Casarini DE, Fortes ZB, et al (2003) NADPH oxidase and enhanced superoxide generation in intrauterine undernourished rats: involvement of the renin-angiotensin system. Cardiovasc Res 59: 767-775.

32. Gao L, Wang W, Li YL, Schultz HD, Liu D, et al. (2004) Superoxide mediates sympathoexcitation in heart failure: roles of angiotensin II and $\mathrm{NAD}(\mathrm{P}) \mathrm{H}$ oxidase. Circ Res 95: 937-944.

33. Griendling KK, Sorescu D, Ushio-Fukai M (2000) NAD(P)H oxidase: role in cardiovascular biology and disease. Circ Res 86: 494-501.

34. Schieffer B, Luchtefeld M, Braun S, Hilfiker A, Hilfiker-Kleiner D, et al. (2000) Role of $\mathrm{NAD}(\mathrm{P}) \mathrm{H}$ oxidase in angiotensin II-induced JAK/STAT signaling and cytokine induction. Circ Res 87:1195-1201.

35. Touyz RM, Berry C (2002) Recent advances in angiotensin II signaling. Braz J Med Biol Res 35: 1001-1015. 